\title{
Naturaleza y Cultura: una dicotomía de límites difusos*
}

\author{
Andrea Milesi *
}

\section{Resumen}

En este trabajo se discute la dicotomía naturaleza/cultura en las dimensiones epistemológica y social. En la dimensión epistemológica, se pone en cuestión una concepción del conocimiento que encuentra su expresión en categorías duales, por caso, naturaleza/cultura. En la dimensión social se analiza la gestación de procesos sociales de resignificación, en la cual la tradicional creencia en la superioridad de la especie, y con ello de la cultura, es puesta en cuestión. La posibilidad misma de continuar pensando a la naturaleza y a la cultura como dominios diferenciados y en relación jerárquica queda desvirtuada.

\section{Palabras clave}

Naturaleza - cultura - categorías duales.

\section{Abstract}

* Artículo recibido el 19 de marzo de 2013. Aceptado el 9 de octubre de 2013.

* Magíster en Antropología Social (IFFCH- UNICAMP). Profesora titular regular cátedra El sujeto desde una perspectiva Socio Antropológica y Cultural. Escuela de Trabajo Social, Universidad Nacional de Córdoba (UNC). Profesora adjunta regular, cátedra Antropología Cultural Contemporánea y Latinoamericana. Facultad de Psicología, UNC.

Contacto: andreaimilesi@gmail.com 
This work discusses the nature / culture dichotomy in epistemological and social dimensions. In the epistemological dimension, it questions a conception of knowledge that finds expression in dual categories, for case, nature / culture. In the social dimension it analyses the gestation of social processes of resignificance, where the traditional belief in the superiority of the human species, and with it of the culture, is challenged. The possibility itself of continue thinking the nature and the culture as differentiated domains and in hierarchic relation loses value.

\section{Keywords}

Nature - culture - dual categories.

\section{Introducción}

En la actualidad asistimos a un proceso de discusión teórica y epistemológica que coloca en duda la validez y eficacia de diversas dicotomías acuñadas en la modernidad, tales como mente/cuerpo, hombre/mujer, naturaleza/cultura.

El auge y prestigio alcanzado por las ciencias naturales en el siglo XIX impactó fuertemente en la configuración del saber. Esto trajo como consecuencia que el marco de desarrollo de la producción científica estuviera dado por la organización del conocimiento distribuido en disciplinas. Esta estructuración colocó a la Antropología en un lugar de privilegio para el tratamiento de los temas que atañen a la cultura, al tiempo que vino a reforzar los límites con los dominios de la naturaleza. De esta forma, la disciplina prácticamente desde sus inicios se interesó por los modos en que los distintos colectivos sociales interactúan con su entorno, pero dentro de los estrechos límites establecidos por la dicotomía naturaleza/cultura.

De todos modos, en las últimas décadas la proliferación de problemas ambientales no solo ha instalado esta cuestión en la agenda política, sino que también ha llevado a una mayor complejización de las producciones teóricas en las ciencias naturales y sociales. La Antropología no ha sido ajena a este proceso. La emergencia de la crisis ambiental ha puesto en evidencia la potencialidad de sus estudios para el tratamiento de la relación entre naturaleza y cultura, con aportes que se extienden tanto a otras ramas del conocimiento, como más allá de los límites estrictamente académicos (Milton, 1995).

Dentro de este contexto, este trabajo propone analizar el tema naturaleza/cultura abarcándolo en sus dimensiones epistemológica y social.

En su dimensión epistemológica, estaría siendo puesta en cuestión una concepción del conocimiento que engloba la forma de clasificar y catalogar que encuentra su expresión en categorías duales; por caso, la dicotomía naturaleza/cultura. 
En su dimensión social es posible señalar la gestación de procesos sociales de resignificación en los cuales se reconocen los impactos negativos de la actividad humana en la naturaleza y las repuestas que esta genera. La tradicional creencia en la superioridad de la especie, y con ello de la cultura, queda desvirtuada al observarse ciertas reacciones de la naturaleza. Más aun, la posibilidad misma de continuar pensando a la naturaleza el ámbito de "lo dado" y a la cultura potestad humana por excelencia de "lo creado", como dominios diferenciados y en relación jerárquica, se coloca en cuestión.

Desde la Antropología, la proliferación de estudios relativos a las relaciones naturaleza, cultura y sociedad, en los cuales las problemáticas ambientales constituyen preocupaciones de primer orden, aportan elementos para poner en duda la adecuación de los abordajes basados en la distinción arriba mencionada.

Sin perder de vista que ambas dimensiones, epistemológica y social, se encuentran íntimamente relacionadas, a los fines analíticos se las presentará de modo desagregado. En primer lugar, se realizará un breve recorrido por las modalidades de tratamiento de la relación naturaleza/cultura a lo largo de la historia de la Antropología, con el objetivo de mostrar los cambios que se han ido gestando en la disciplina. Luego, se aborda la problemática ambiental en tanto fenómeno complejo que impacta en los colectivos sociales y su expresión en las producciones académicas. Establecido este marco, se analizará el aspecto epistemológico de la cuestión: esto es, el grado de adecuación de la dicotomía naturaleza/cultura, en tanto construcción teórica disociadora, a la luz de la problemática ambiental actual.

\section{Enfoques de la Antropología sobre la relación naturaleza/cultura}

A lo largo de su historia, la Antropología se ha preocupado por analizar las relaciones de los distintos colectivos sociales con su entorno natural. La magnitud de estos trabajos dio origen a un área específica: la Antropología Ecológica, que cuenta con algo más de cien años de trayectoria.

El término ecología, acuñado por el biólogo alemán Ernst Haeckel, hace referencia al estudio de los distintos organismos en su "oikos" (casa- hábitat-ambiente-medio). La Antropología Ecológica toma este nombre para dar cuenta de las relaciones entre grupo humano, cultura y medio natural. La preocupación abarca procesos migratorios y de supervivencia, como así también las transformaciones ambientales, económicas y sociales; los impactos en las actividades productivas y organizacionales; los aspectos psicológicos y las representaciones sociales, etc., siempre con la idea de considerar la interacción permanente y necesaria de la naturaleza y la cultura. Desde finales de los 70 cobra fuerza la Antropología Ambiental (para algunos autores, variante particular dentro de la Antropología Ecológica: Montoya, 1993; Durand, 2002), que coloca el acento en situaciones de crisis ambiental, sea por mal manejo de los recursos, disputas 
de territorios, modalidades de apropiación cultural del ambiente por los colectivos sociales, etc.

En su desarrollo histórico, la Antropología Ecológica presenta tres modalidades principales de tratamiento de la cuestión, caracterizadas por fluctuaciones entre distintos determinismos que avanzan hacia perspectivas integradoras (Milton, 1995).

Una primera versión la ofrece el llamado "determinismo ambiental". Estos estudios sustentan un condicionamiento del medio sobre la vida social y cultural del hombre. Por ejemplo, han observado una vinculación categórica entre el ambiente y las tecnologías por emplearse (Mason, 1896). Dentro de esta perspectiva se considera que los factores ambientales inciden directamente sobre los comportamientos de los colectivos humanos, quienes deben adaptarse a su entorno, y por tanto sus acciones son determinadas por el medio en que desarrollan su existencia. Algunos estudios encuentran en el clima el principal factor explicativo del comportamiento humano, hallando las posibilidades de desarrollo de las culturas necesariamente ligadas a la su benignidad (Huntington, 1924).

En la misma línea, una versión más moderada conocida con el nombre de "posibilismo" veía en el ambiente más que una determinación ineludible un límite a las posibilidades de desarrollo social y cultural de los grupos humanos. Así, por ejemplo, la prolongación de un período de sequía podría afectar las estrategias de desplazamiento de grupos cazadores, pero no implicaba que necesariamente estos cambios debieran ocurrir.

Otra de las propuestas es la ofrecida por el "determinismo cultural". El acento se traslada a los colectivos sociales que en sus procesos de adaptación impactan en el entorno. Así, los procesos culturales pasan a ser los determinantes de la dinámica ambiental. Este tipo de abordaje entiende que el entorno podría contribuir a explicar ciertos rasgos culturales, pero en modo alguno es considerado como un factor determinante. Por ejemplo, sobre un mismo espacio físico, grupos culturales diferentes establecerían estrategias diferenciales de relación con el ambiente, con lo que el ambiente, en realidad, constituirá un límite a las actividades y comportamientos dentro de un abanico de opciones posibles (Ellen, 1982; Rusch, 1984).

Finalmente encontramos los "análisis ecosistémicos". En esta perspectiva el ambiente natural constituye un factor de creatividad que ejerce una presión selectiva sobre la cultura. El acento se coloca en la interacción entre el ámbito de la cultura y el del medio natural. El empleo del concepto de ecosistema rescata la idea de interconexión estable entre cultura, biología y medio ambiente. Desde esta mirada, la especie humana es una más entre la enorme variedad de especies biológicas en el planeta, siendo sus interacciones con el medio físico y biológico continuas, indisolubles y necesarias (Durand, 2002, p. 175). Un ejemplo de este tipo de perspectivas lo 
encontramos en un célebre trabajo de Roy Rappaport ${ }^{1}$, quien explica cómo en Nueva Guinea un ritual de tributo a los ancestros, en el cual se llevaba a cabo el sacrificio de cerdos, colocaba en estrecha relación la satisfacción de las creencias místicas del grupo social y el control de la competencia por el alimento. Cuando los cerdos se multiplicaban más allá de los límites tolerables, era necesario sacrificarlos, ya que competían con el grupo humano por los granos y los espacios destinados a las plantaciones. Este tipo de análisis, al recuperar las interrelaciones de los humanos con su ambiente y la manera en que se condicionan mutuamente, pone al descubierto un mecanismo de equilibrio de las relaciones entre el ecosistema y la población.

Las posiciones hasta aquí reseñadas presentan la particularidad de mantener la dicotomía naturaleza/cultura. Actualmente nos encontramos con tendencias que cuestionan esta dicotomía. Más interesante aun: ponen al descubierto cuánto tiene de cultural pensar la realidad desde esta dicotomía y de qué modo esto correspondería a una construcción cultural de Occidente, que no se registra en otras formaciones culturales.

Actualmente, en un contexto donde la naturaleza se muestra fuertemente impactada por la actividad humana, surgen importantes producciones teóricas que desde diversas ramas del conocimiento reflexionan acerca de la relación naturaleza /cultura abriendo un abanico de posibilidades para su tratamiento, entre las que se destaca el propio cuestionamiento de esta dicotomía.

La práctica dominante en las ciencias sociales procede considerando a los seres humanos como situados en el vacío como si la satisfacción de sus necesidades no obligara a utilizar, manipular y transformar la naturaleza, como si sus decisiones no tuvieran impactos muchas veces decisivos sobre ella. La mayoría de las ciencias sociales son tributarias de la ilusión metafísica que inundó la modernidad y que separó al ser humano de la naturaleza, generando una ficción antropocéntrica que aún persiste entre pensadores y las corrientes más avanzadas de la ciencia contemporánea. (Toledo y González de Molina, 2007, p. 85)

La concepción acerca de lo que es natural deviene de la propia definición que construimos sobre la naturaleza, pero esta mirada es culturalmente mediada. En otros términos: toda visión sobre la naturaleza presupone una construcción cultural. Pero al mismo tiempo, aquellas nociones que permean nuestro cotidiano a pesar de formar parte de construcciones culturales particulares frecuentemente son vividas, pensadas y sentidas, como fácilmente extensibles a los distintos colectivos humanos, que, cuando presentan actitudes, comportamientos o valoraciones diferentes de las propias, nos

\footnotetext{
${ }^{1}$ Se trata del libro titulado Cerdos para los ancestros. El ritual en la ecología de un pueblo de Nueva Guinea, cuya primera edición data de 1968.
} 
llevan a reflexionar acerca de lo relativo de nuestras concepciones. En alguna medida naturalizamos nuestras construcciones.

El aprendizaje que cada sociedad desarrolla en interacción con su ambiente va a conformar en los sujetos una particular percepción de su entorno. La mirada que construyen los actores sociales de su medio se traduce en escalas que asignan valores diversos al entorno en que los sujetos desarrollan su existencia, como así también en las representaciones, comportamientos y apropiaciones respecto del ambiente.

Analizar el tema ambiental desde la perspectiva de las representaciones sociales (Ibáñez, 1988) permite recuperar la importancia de los significados, el papel de los aspectos simbólicos y de la actividad interpretativa de las personas, otorgándoles un lugar destacado a las condiciones sociales, económicas, políticas y culturales en que se encuentran los actores sociales, ya que estas serán relevantes para la lectura que realizan del ambiente. Esto no agota la cuestión, ya que no necesariamente las representaciones constituyen fiel reflejo de los fenómenos que refieren. Es decir, los problemas ambientales generados por el desarrollo particular de nuestra sociedad existen objetivamente, aunque no se los perciba en toda su magnitud ${ }^{2}$.

\section{De la naturaleza al ambiente}

Todo período histórico comparte formaciones inconscientes que condicionan y estructuran los conocimientos (Foucault, 1985, 1997). Cada época, con sus formas de ver y expresar, va conformando un perfil particular, proporcionando un tono que permite establecer límites y distinciones puestas de manifiesto en los discursos que circulan. Al ser todo discurso un producto social, no es posible pensarlo fuera de las condiciones de su producción, lo que involucra aspectos ideológicos, culturales, institucionales, y las posibilidades mismas de los actores de expresarse dentro de las particulares condiciones históricas en que se encuentran. El discurso sobre la naturaleza no escapa a esta regla.

La concepción antropocentrista de la vida, que toma al ser humano como la medida de todas las cosas, hegemónica desde la modernidad, fue determinante para la relación del hombre con su medio. Realizando un recorrido acerca de las concepciones sobre la naturaleza a lo largo de la historia, María Zeballos de Sisto muestra de qué modo, en lo que conforma el mundo occidental, gradualmente se impondría la idea de que "... le corresponde al hombre, ordenar, usar y gozar del entorno natural y sus recursos. Este

\footnotetext{
${ }^{2}$ En la provincia de Córdoba, Argentina, la superficie total del bosque nativo pasó de 16.532 .100 a 594.000 ha. Este es un hecho concreto y verificable, que existe más allá que los colectivos sociales, por caso, la sociedad cordobesa en su conjunto o parte de ella, lo conozcan y aun conociéndolo lo consideren un problema (Barchuk, A. H.; Britos A. H. y De la Matta E. 2009. Diagnóstico de la cobertura de bosques nativos de la provincia de Córdoba en Sistema de Información Geográfico).
} 
uso y goce admite un único límite: el que imponen los principios técnicos y económicos" (Zeballos de Sisto, 1992, p. 144).

El antropocentrismo toma a la naturaleza como una entidad externa, un objeto, un recurso destinado a satisfacer las necesidades humanas, susceptible de apropiación, modificación, control, transformación, en fin, cualquier actividad que decida imponerle la razón humana. Razón considerada capaz de construir los conocimientos necesarios que le permiten colocarse por encima de todo lo existente, creando y recreando, tantas veces como sea necesario, el mundo material.

Esta mirada que sitúa al ser humano como centro de todo lo que lo circunda y fin absoluto de la naturaleza, asociada a la concepción propia del sistema capitalista, donde el beneficio económico ocupa el primer lugar en el esquema de prioridades, resulta en una combinación fatal para la vida del planeta.

La multiplicación y profundización de los problemas ambientales derivados del mal manejo de los suelos, de la contaminación del aire y del agua, de la disminución y desaparición de especies animales y vegetales, de los cambios en el clima, del crecimiento no planificado de los centros urbanos, de considerables aumentos de la población mundial fueron conformando señales de alerta ${ }^{3}$.

La década del 60 aparece como el momento de despegue de las preocupaciones relacionadas con los impactos negativos derivados de la actividad humana sobre la naturaleza. La preocupación por este tema toma formas definidas, primero focalizadas en problemáticas puntuales (contaminación del agua, uso de pesticidas, etc.), avanzando hacia miradas más abarcativas (calidad de vida en general) en los Estados Unidos, Canadá, Japón, Nueva Zelandia, Europa Occidental, Australia. En algunos sectores, fundamentalmente académicos, comenzó a gestarse una conciencia acerca de las limitaciones que debían imponerse a los hallazgos científicos, interesados exclusivamente en el rédito económico y sin la adecuada ponderación del empleo de métodos, técnicas, sustancias y descubrimientos para la vida misma.

El trabajo de Rachel Carson Primavera Silenciosa, publicado en 1962, constituye un referente histórico de relevancia. En ese texto, que en su momento causó grandes controversias, la autora advertía acerca del empleo indiscriminado de sustancias químicas peligrosas (como el $\mathrm{DDT}^{4}$ ) para los distintos tipos de vida en la tierra. Realizaba una enfática llamada de atención a la acción humana sobre la naturaleza.

En ese período comienza a perfilarse un discurso renovado acerca de la naturaleza, que entre otros aspectos cuestionará las posibilidades de la ciencia para controlar los estragos planetarios.

\footnotetext{
${ }^{3}$ Desde la emblemática reunión de 1968 en Roma, donde posteriormente surgirá el alarmante informe de Dennis Meadows, pasando por las distintas Cumbres de la Tierra (Estocolmo 1972, Río de Janeiro 1992, Johannesburgo 2002, Rio+20 2012), los informes aportados evidencian un progresivo incremento de las problemáticas ambientales, sin que se avizoren compromisos serios para revertirlas.

${ }^{4}$ Siglas del potente insecticida compuesto de dicloro, difenil, tricloroetano.
} 
En 1972 se celebra en Estocolmo la Conferencia de las Naciones Unidas sobre el Medio Ambiente Humano, jalón cardinal en la historia ambiental, la que contó con la asistencia de representantes de unos ciento treinta países. En esa oportunidad la cuestión ambiental logró alcanzar a los centros de poder. En los dominios donde tradicionalmente imperaba un discurso basado en la idea de "al mundo natural hay que transformarlo", comenzó a manifestarse, al menos discursivamente, la idea de "al mundo natural hay que conservarlo". La naturaleza comenzó a abandonar su carácter inagotable para perfilarse como un bien limitado. Por otra parte, los problemas generados a partir de la relación del ser humano con el ambiente, como el uso del agua, las alteraciones climáticas, la pérdida de biodiversidad, el debilitamiento progresivo de la capa de ozono, la contaminación del aire, el crecimiento de la población, la falta de energía, el empobrecimiento de los recursos naturales, en general, dejaron de ser cuestiones apenas limitadas a un reducido círculo, para alcanzar cierta masividad, al menos en el discurso. Esto es, salieron las cuestiones ambientales del ámbito estrictamente académico y científico provocando un paulatino crecimiento en su difusión a un público no especializado. Un nuevo discurso comienza a perfilarse: el de la preservación. También surge el de la resistencia a un desarrollo ilimitado y sin control. Desde la sociedad civil, se sumaron nuevos actores que comenzaron a disputar un espacio por controlar su calidad de vida, su derecho a elegir el ambiente en que deseaban vivir.

En los 80 la llamada de atención se expande al resto del planeta. El tema es abordado en América Latina, Europa Oriental, Asia, etc.

Este fenómeno que venimos comentando causó fuerte impacto dentro de las ciencias sociales. La emergencia de los movimientos ambientalistas en el mundo, no apenas por su número sino también por su trascendencia, llevó a que se constituyan en tema de interés entre los investigadores, pudiendo señalarse dos grandes tendencias. Por un lado, y especialmente en Europa, los estudios se orientaron al análisis del ambientalismo en términos de su potencial social en larga escala y posibilidades de generación de cambios históricos. Por otro lado, y con base principalmente en Estados Unidos, las investigaciones se dedicaron más al análisis de las características organizacionales del movimiento. Aunque debe destacarse la fuerte tendencia a, paulatinamente, dar cuenta de todos los aspectos antes mencionados (Benton/Redcliff, 1994).

En el caso de Latinoamérica, los trabajos presentan una fuerte preocupación por recuperar las categorías nativas, los conocimientos ecológicos de campesinos y grupos indígenas, su papel en la transformación y construcción de sus entornos, como así también las articulaciones entre los movimientos sociales y las políticas ambientales ${ }^{5}$.

\footnotetext{
${ }^{5}$ Es el caso de los trabajos de Arturo Escobar, Astrid Ulloa, Eduardo Viveros de Castro, entre otros.
} 
Lo que resulta importante destacar es que la nota común a estas perspectivas es el desplazamiento de aquellas ideas de naturaleza como entidad externa y pasiva, en dirección a las posturas que le reconocen un papel activo. Un ejemplo emblemático de esta miradas lo ofrece la famosa y controvertida Hipótesis Gaia ${ }^{6}$ acuñada por James Lovelock, para quien la naturaleza, lejos de ser apenas un soporte material para la vida, es considerada una suerte de organismo viviente con capacidad de reacción frente a distintos estímulos, entre otros el comportamiento de la especie humana. Un espacio vital con leyes propias que no podrían ser alteradas o transgredidas sin tener en cuenta las consecuencias de estos comportamientos. Escribe Lovelock:

Al ecologista que le gusta creer que la vida es frágil y delicada y que está en peligro por la brutalidad humana, no le gusta lo que ve cuando observa el mundo a través de Gaia (la tierra). La damisela en peligro a la que esperaba salvar resulta ser una madre metida en carnes y robusta, devoradora de hombres. (Lovelock, 1992, p. 93)

Dicho autor llegó a sugerir, inclusive, que la verdadera función del hombre, tanto como la de los demás mamíferos, era la de constituir el medio ideal para la vida de millones de bacterias que habitaban en su organismo.

En este marco puede observase la transformación en la percepción del entorno y cómo progresivamente la relación social con este cobra relevancia. Este cambio en la forma de ver la relación del ser humano con la naturaleza también se manifiesta en las formas de expresión. Paulatinamente en los discursos fue sustituyéndose el término "naturaleza" por el de "medio ambiente" (el más corriente) o simplemente "ambiente".

Los discursos sobre la naturaleza/ambiente reflejan un desplazamiento de visiones antropocéntricas a visiones biocéntricas: todo ser vivo merece consideración. El ser humano deja de poseer un valor diferenciado y superior frente al resto de la creación, es parte de la naturaleza, se coloca el acento en la vida misma. Los axiomas de la sociedad occidental, antropocentrismo y creencia en el progreso ilimitado, son puestos en cuestión. El interjuego de relaciones entre medio físico y actividad humana pasa a formar parte del paisaje. Se proclama la necesidad de atender a las dinámicas con que tienen lugar los procesos. Las interrelaciones y la mutua interdependencia entre los ecosistemas y las condiciones sociales, económicas y culturales aparecen como la clave para abordar la cuestión.

Hay una coincidencia importante dentro de la Antropología, en el sentido de que las representaciones que construyen los distintos grupos sociales respecto de su entorno está relacionada directamente con la forma en que usan y viven en su ambiente, por lo que no "... debería sorprendernos que los conceptos de naturaleza en

\footnotetext{
${ }^{6}$ Gaia, nombre que utilizaban los griegos para designar a la Tierra, sería una entidad comprensiva del planeta entero, con la capacidad de regular su clima y su composición, de suerte que la biosfera sería en realidad producto de la interacción de todos los fenómenos vivos constituyendo una totalidad autorregulada.
} 
las sociedades industriales sean complejos y ambiguos, tal como cabe esperar que ocurra en toda sociedad cuyos miembros se relacionan con su entorno de modos muy variados" (Milton, 1995).

En este contexto se observan diferencias importantes en las percepciones. Posicionamientos que oscilan entre la tragedia y el colapso inevitable -estamos gestando nuestra destrucción - y la euforia confiada en los descubrimientos tecnológicos - la ciencia al servicio de la preservación es la clave- dan cuenta del ambiente en crisis, y consecuentemente de las prácticas que seguir.

Los discursos se reparten entre evaluaciones y procedimientos fragmentados, por un lado, y planteos integrales, por el otro.

Con diferentes denominaciones, pueden observarse formulaciones que tienen como marco la idea de una modernidad ecosostenible, donde el acento recae en el tratamiento y solución de problemas ambientales específicos. Por ejemplo, las regulaciones en torno al empleo de plaguicidas; las políticas de manejo de los recursos hídricos, el impulso al reciclado de materiales, las prácticas de concientización de los consumidores para que se inclinen por productos "ambientalmente amigables", etc.

Frente al tipo de propuestas arriba reseñadas, surgen los posicionamientos que impulsan una mirada holística de la problemática, considerando la necesidad de discutir políticas ambientales globales. Para Astrid Ulloa la clave de diferenciación está en considerar la necesidad de un cambio que incluya o no las cuestiones sociales, lo que abarcaría no apenas los problemas económicos y políticos, sino también las responsabilidades diferenciales de los distintos sectores y actores involucrados (Ulloa, 2007).

Otros abordajes igualmente abarcativos los constituyen el ecofeminismo (en sus diversas variantes: radical, cultural, liberal, socialista, etc. $)^{7}$; la ecología profunda ${ }^{8}$ o los mismos biorregionalismos, que presentan como eje central de sus propuestas la necesidad de la adecuación de las políticas públicas y demás normativas del ambiente en que serán aplicadas. A través de diversas formulaciones, las propuestas holísticas confluyen en el sostenimiento de implementar una nueva racionalidad ambiental tendiente a la equidad, que respete la diferencia y preserve la diversidad social, cultural y ambiental. En este orden, las propuestas de integración de los saberes locales - de las que por otra parte diversos gobiernos dicen hacerse eco- dan batalla sostenida frente a las contradicciones que presentan modelos de desarrollo altamente depredadores.

\footnotetext{
${ }^{7}$ Dentro de estas corrientes encontramos figuras célebres como Vandana Shiva y Wangari Maathai, para quienes más allá de posiciones particulares, no se puede hablar de desarrollo sostenible si no se contemplan los derechos humanos y en especial los derechos de las mujeres.

${ }^{8}$ Iniciado en los 70 por Arne Naes fue creciendo, conformando en la actualidad un movimiento que básicamente sostiene la necesidad de una integración total del ser humano con la naturaleza. En esta línea se destacan los trabajos de George Session y Bill Devall; Fritjof Capra y otros.
} 


\section{Los difusos límites de una dicotomía precisa}

Entrando a considerar los aspectos epistemológicos de la dicotomía naturaleza/cultura, cabe destacar que el notable desarrollo alcanzado por las ciencias de la naturaleza en el siglo XIX las llevó a que se constituyan en el modelo científico por seguir. Consecuentemente, el conocimiento fue compartimentado en áreas específicas, y en esta estructuración la Antropología como disciplina quedó a cargo principalmente de los aspectos culturales. Al separar la naturaleza de la cultura, y con ello de la sociedad, la modernidad las consideró como dos categorías claramente diferenciadas. Progresos posteriores en la teoría antropológica han llevado a replantear la reproducción y naturalización de las diversas dicotomías acuñadas en la modernidad, tales como mente/cuerpo, hombre/mujer, naturaleza/cultura, como instrumentos adecuados para analizar la realidad. En este contexto y a la luz de las problemáticas ambientales que se multiplican se puede observar cómo los estudios progresivamente vienen a poner en cuestión los contenidos de cada una de las categorías de naturaleza y de cultura, al menos tal como vienen siendo empleadas desde el siglo XIX, como ámbitos diferenciados y jerárquicamente relacionados.

Philippe Descola considera que en las sociedades occidentales el Naturalismo constituye el modo de identificación típico. La sociedad humana es tomada como modelo, la cultura es lo que se diferencia de la naturaleza. Entre humanos y no humanos la diferencia básica residiría en la conciencia reflexiva, la subjetividad, el poder significar, el dominio de los símbolos y el lenguaje por medio del cual esas facultadas se expresan. Específicamente, el Naturalismo se caracteriza por crear

un dominio ontológico específico, un lugar de orden y necesidad donde nada ocurre sin una razón o una causa ( ... ) permea tanto nuestro sentido común como nuestra práctica científica ( ... ) estructura nuestra epistemología y en particular nuestra percepción de otros modos de identificación (Descola, 2001, pp. 108, 109).

De esta manera, en una clara muestra de etnocentrismo, se extiende a todas las sociedades humanas la oposición naturaleza /cultura.

Por el contrario, Descola sostiene que todas las sociedades establecen algún tipo de compromiso entre naturaleza y cultura, por lo que cabría entonces examinar las diversas expresiones particulares. Al proponerse este tipo de abordajes, se traslada el acento a los procesos, cobra relevancia la distinción entre aquellos colectivos sociales que ven los mundos humanos y no humanos como un continuo, y aquellos que los consideran como ámbitos independientes. La dicotomía naturaleza/cultura, fundamento clave de la epistemología modernista, es así claramente superada.

Ir más allá del dualismo abre un paisaje intelectual completamente diferente, un paisaje en el que los estados y las sustancias son sustituidos por procesos y relaciones; 
la cuestión más importante ya no es cómo objetivar sistemas cerrados, sino como explicar la propia diversidad de los procesos de objetificación. (Descola, 2001, p. 23)

La oposición antes clara y firme entre naturaleza/cultura (y también cuerpo/mente, emoción/razón, mujer/hombre, acción/pensamiento) es puesta en duda. Los límites antes precisos se tornan difusos. Cuando se intenta encontrar su correlato social, estas categorías epistemológicas presentan dificultades en su aplicación. Este prototipo constituyente del quehacer científico entra en debate, sospechándose su eficacia para dar cuenta de nuestro mundo.

Bruno Latour (2007) va más allá en este cuestionamiento llegando a afirmar que la representación del mundo de nuestra sociedad moderna, donde la naturaleza y la cultura resultan irremediablemente opuestas, no encuentra correlato en la práctica. Por el contrario, la creación de híbridos, esto es, objetos que conjugan naturaleza y cultura, ha sido una constante a lo largo de la historia. Esta situación silenciada, por veces negada, opera como garantía de superación. En ese sentido, dirá este autor que nunca fuimos verdaderamente modernos. De allí la necesidad de buscar alternativas epistemológicas que permitan considerar ambos órdenes — naturaleza y cultura- de modo simétrico, y lejos de separarlos, los integren en un continuo.

Las discusiones en torno a la reconsideración de estas categorías acarrean consecuencias no apenas teóricas, sino también políticas, en las que operan procesos de resignificación, negociación y conflicto. Todo ello porque las categorías de naturaleza y cultura operan en la sociedad. Cuando entran a considerarse grupos sociales en sus particulares contextos, se observa cuánto de los contenidos de esta dicotomía son resultado de un proceso histórico-político específico, atravesado por relaciones desiguales de poder. Pongamos por caso las explotaciones mineras a cielo abierto. Tenemos por un lado fuertes corporaciones multinacionales (respaldadas por una legislación abiertamente permisiva) y por el otro pequeñas comunidades alejadas de los centros urbanos, con recursos limitados. La resistencia que llevan a cabo las asambleas ciudadanas a este tipo de explotaciones altamente contaminantes está claramente signada por la asimetría. Al definir el Estado, un espacio territorial como recurso natural, se traduce en la práctica en la posibilidad de disponerlo para ser negociado. Por su parte, las comunidades que resisten a la megaminería definen al espacio territorial como bien común, lo que limita la posibilidad de negociarlo, o la posibilidad de emplearlo de modo que se afecte negativamente la vida. Se observa una trayectoria que da cuenta de transformaciones que van desde concepciones de la naturaleza como entidad apolítica a construcciones sociales con implicaciones políticas (Ulloa, 2007).

De cara a los múltiples conflictos ambientales existentes en la actualidad, puede observarse cómo las luchas ambientales que llevan a cabo distintos colectivos sociales dan cuenta de una particular apropiación cultural del ambiente. Estos cambios en las 
concepciones tienen sus consecuencias políticas observables en las resignificaciones que otorgan los colectivos sociales a los ambientes en que participan. Las representaciones sociales conformadas en torno a esta cuestión ofrecen modelos orientadores de las prácticas, donde la recategorización del medio, la redefinición de la naturaleza, que en no pocos casos alcanza contornos místicos, llevan a que la separación humano/no humano en órdenes jerárquicos diferenciados no tenga cabida.

\section{Consideraciones finales}

Resulta relevante reflexionar en torno a los modos estructurar y organizar el conocimiento, desde que al clasificar y categorizar establecemos el elenco de elementos importantes y necesarios que deben ser considerados, y el modo adecuado de llevar adelante la tarea. Reconsiderar estas cuestiones, procurando relacionarlas con los fenómenos de la vida en sociedad - por caso, los problemas ambientales- nos coloca en alerta frente a los procesos de naturalización de los que somos artífices y víctimas a un mismo tiempo.

Aprieten el aerosol más inocente y se verán llevados hacia la Antártida, y de ahí hacia la Universidad de California en Irvine..., las cadenas de montaje en Lyon, la química de los gases inertes, y de ahí quizás hacia la ONU, pero ese hilo frágil será roto en otros tantos segmentos cuantas disciplinas puras hay... (Latour, 2007, p. 17)

Esta es la estrategia de la modernidad: purificar los fenómenos hasta ubicarlos o bien en la naturaleza o bien en la cultura. Pero ocurre que nuestro cotidiano ha estado siempre poblado de objetos híbridos.

La creencia en la existencia de esferas claramente delimitadas entre la naturaleza y la cultura no permite advertir el tránsito permanente entre ambos dominios, que al final de cuentas no estarían tan diferenciados, desde que habitamos un mundo donde naturaleza y cultura en nuestro cotidiano se conjugan dando origen a universos indiferenciados.

Hablar de ambiente pone en evidencia la interrelación necesaria entre naturaleza y cultura. Descubre una de las tantas combinaciones que permean nuestra sociedad, donde la pretensión de clasificar, categorizar y establecer límites precisos se vuelve cada vez más impracticable, situación que necesariamente recogen las producciones teóricas que reclaman por formulaciones más flexibles que permitan pensar y comprender la sociedad en que vivimos, donde las seguridades que ofrecían los contornos precisos de las dicotomías de la modernidad abandonan su exactitud evidenciando ahora sus límites difusos. 


\section{BIBLIOGRAFÍA}

Benton, T. Y M. Redclift (1994). Social Theory and de Global Enviroment. Londres: Routtledge.

Descola, P. (2001). Construyendo Naturalezas, Ecología Simbólica y Práctica Social, en Naturaleza y Sociedad Descola P. Y Pálsson G. (coord.) Perspectivas antropológicas. México: Siglo XXI ed.

Descola P. (2012) Más allá de naturaleza y cultura. Buenos Aires. Argentina: 1ra. ed., Amorrortu.

Dobson, A. (1997). Pensamiento Político Verde. Barcelona: Paidós.

Durand, L. (2002). La relación ambiente cultura en Antropología: recuento y perspectivas en Nueva Antropología. Revista de Ciencias Sociales, ISSN 0185-0636, N. ${ }^{\circ}$ 61.

Ellen, R. (1982). Environment, Subsistence and System: the ecology of small scale social formations. Cambridge: Cambridge University Press.

Ellen, R. (2001). La geometría cognitiva de la naturaleza. Un enfoque contextual. en Naturaleza y Sociedad Descola P. Y Pálsson G. (coord.) Perspectivas antropológicas. México: Siglo XXI ed.

Foucault, M. (1985). Las palabras y las cosas. España: Planeta Agostini. Foucault, M. (1997). La arqueología del saber. México: Siglo XXI.

Garrido, F. González De Molina, M. Serrano, J. Y Solana, J. (eds) (2007). El paradigma ecológico en las ciencias sociales. Barcelona: Icaria.

Huntington, E. (1924). Civilization and Climate. New Haven: Yale University Press.

Ibáñez, T. (1988). Ideologías de la vida cotidiana. Barcelona, España: Sendai.

Latour, B. (2007). Nunca fuimos modernos. Ensayo de antropología simétrica. Argentina: Siglo XXI ed.

Lovelock, J. (1992). Gaia. Un modelo para la dinámica planetaria y celular en W.I.Thompson (ed) Gaia. Implicaciones de la nueva biología. Barcelona: Kairos (2da. ed.).

Mason, O. (1896) 'Influence of Environment upon Human Industries or Arts.' Annual Report of the Smithsonian Institution for 1895: 639-65, Washington.

Milesi, A. (1997). La Percepción social de la Problemática Ecológica en Ciencias Sociales Publicación del Área de Ciencias Sociales del CIFFyH, UNC, N. ${ }^{\circ} 1$, 1997. ISSN 1515-1859.

Milesi, A. (2009). Formaciones discursivas sobre el ambiente. ALEDar -. www.fl.unc.edu.ar/aledar/index.php

Milton, K. (1995). Ecologías: antropología, cultura y entorno. www.unesco.org/issj/rics154/miltonspa.html

Rutsch, M. (1984) El relativismo cultural. México. Línea. 
Toledo, V. Y González De Molina, M. (2007). El metabolismo social: las relaciones entre la sociedad y la naturaleza en El paradigma ecológico en las ciencias sociales $\mathrm{F}$. Garrido, M. González de Molina, J. L. Serrano y J. L. Solana (eds.), Barcelona: Icaria.

Ulloa, A. (2004). La construcción del nativo ecológico: complejidades, paradojas y dilemas de la relación entre los movimientos indígenas y el ambientalismo en Colombia. Bogotá, Colombia: Instituto Colombiano de Antropología e Historia -ICANH.

Zeballos De Sisto, M. (comp.) (1992) Sociedades Humanas y Equilibrio Ecológico. Buenos Aires: Letra Buena. 